\title{
COMPOSITION OPERATORS INDUCED BY RATIONAL FUNCTIONS
}

\author{
R. K. SINGH
}

\begin{abstract}
A necessary and sufficient condition for a rational function to define a composition operator on $L^{p}(\mu)$ is given in this paper, where $\mu$ is the Lebesgue measure on the Borel subsets of the real line. In particular, all polynomials inducing composition operators are completely determined.
\end{abstract}

1. Preliminaries. Let $(X, \mathcal{S}, \lambda)$ be a $\sigma$-finite measure space and $\phi$ be a measurable transformation on $X$ into itself. Then we (roughly) define a linear transformation $C_{\phi}$ on the Banach space $L^{p}(\lambda)(p \geqslant 1)$ into the space of all complex valued functions on $X$ by $C_{\phi} f=f \circ \phi$. If $C_{\phi}$ is continuous with range in $L^{p}(\lambda)$, then we call it a composition operator on $L^{p}(\lambda)$ induced by $\phi$. The following theorem will be needed.

THEOREM 1. Let $\phi$ be a measurable transformation on $X$ into itself. Then $C_{\phi}$ is a composition operator on $L^{p}(\lambda)$ if and only if there exists an $m>0$ such that $\lambda \phi^{-1}(E) \leqslant m \lambda(E)$ for every measurable set $E$.

Proof. Suppose $C_{\phi}$ is a composition operator on $L^{p}(\lambda)$ i.e. $C_{\phi}$ is bounded. Let $E$ be any measurable set. If $\lambda(E)$ is not finite, then the inequality follows trivially. If $\lambda(E)<\infty, X_{E}$ (the characteristic function of $E$ ) is in $L^{p}(\lambda)$ and

$$
\lambda \phi^{-1}(E)=\left\|C_{\phi} X_{E}\right\|^{p} \leqslant\left\|C_{\phi}\right\|^{p}\left\|X_{E}\right\|^{p}=\left\|C_{\phi}\right\|^{p} \lambda(E) .
$$

Letting $m=\left\|C_{\phi}\right\|^{p}$, we get $\lambda_{\phi}{ }^{-1}(E) \leqslant m \lambda(E)$.

Conversely, suppose there is an $m>0$ such that $\lambda \phi^{-1}(E) \leqslant m \lambda(E)$ for every measurable set $E$. Then the measure $\lambda \phi^{-1}$ is absolutely continuous with respect to $\lambda$. Let $f_{0}$ denote the Radon-Nikodým derivative of $\lambda \phi^{-1}$. Then

$$
\int_{E} f_{0} d \lambda=\lambda \phi^{-1}(E) \leqslant m \lambda(E)=\int_{E} m d \lambda .
$$

Therefore $f_{0} \leqslant m$ (a.e.). Let $f \in L^{p}(\lambda)$. Then

$$
\int|f \circ \phi|^{p} d \lambda=\int|f|^{p} d \lambda \phi^{-1}=\int|f|^{p} f_{0} d \lambda \leqslant m\|f\|^{p} .
$$

This shows that $C_{\phi}$ is bounded. This completes the proof of the theorem.

In this paper we are interested in studying composition operators in a special case. We take $X$ to be equal to $R$, the set of real numbers and we take $\mu$ to be the Lebesgue measure on the Borel subsets of $R$. Now $\phi$ is a measurable (Borel) real valued function on $R$. The following theorem gives a

Received by the editors September 19, 1975.

AMS (MOS) subject classifications (1970). Primary 47B99; Secondary 47B99.

Key words and phrases. Rational functions, composition operators, polynomials, derivative. 
necessary condition for a function $\phi$ to induce a composition operator on $L^{p}(\mu)$.

THEOREM 2. Let $\phi$ be a real valued measurable function on $R$ which is continuous in a neighbourhood of every point where its derivative exists. Then $C_{\phi}$ is a composition operator on $L^{p}(\mu)$ only if $\phi^{\prime}$ is nonzero wherever it exists. ( $\phi^{\prime}$ denotes the derivative of $\phi$.)

Proof. Suppose $C_{\phi}$ is bounded. Then by Theorem 1 there exists an $m>0$ such that $\mu(E) / \mu \phi^{-1}(E) \geqslant 1 / m>0$ for every Borel set $E$. Let $a_{0}$ be a point of $R$ where $\phi^{\prime}$ exists and let $N$ be a neighbourhood of $a_{0}$ on which $\phi$ is continuous. Then it is possible to choose a sequence $\left\{a_{n}\right\}$ from $N$ such that $a_{n}>a_{0}$ for every $n, a_{n}$ tends to $a_{0}$ and $\phi$ has a maximum (or minimum) at $a_{n}$ with respect to the interval $\left[a_{0}, a_{n}\right]$. If we take $E_{n}$ as the interval $\left[\phi\left(a_{0}\right)-\right.$ $\left.\left|\Delta \phi_{n}\right|, \phi\left(a_{0}\right)+\left|\Delta \phi_{n}\right|\right]$, where $\phi_{n}=\phi\left(a_{n}\right)-\phi\left(a_{0}\right)$, then by the above inequality we get $\mu\left(E_{n}\right) / \mu \phi^{-1}\left(E_{n}\right) \geqslant 1 / m>0$ for every $n$. This implies that

$$
2\left|\Delta \phi_{n}\right| / \Delta a_{n} \geqslant 1 / m>0
$$

where $\Delta a_{n}=a_{n}-a_{0}$. From this inequality it is clear that $\left|\phi^{\prime}\left(a_{0}\right)\right| \geqslant 1 / 2 m>$ 0 . This finishes the proof of the theorem.

Note. The condition in the theorem is not sufficient because the derivative of the function $\phi(x)=1 / x^{2}$ is nonzero, but it does not define a composition operator.

The above theorem provides us many examples of functions which do not induce composition operators on $L^{p}(\mu)$. In the following theorem we examine the suitability of monotone functions for inducing composition operators on $L^{p}(\mu)$.

THEOREM 3. Let $\phi$ be a monotone real valued function on $R$. Then $C_{\phi}$ is a composition operator on $L^{p}(\mu)$ if and only if $1 / \phi^{\prime}$ is essentially bounded. In case $1 / \phi^{\prime}$ is essentially bounded, $\left\|C_{\phi}\right\|^{p}=\left\|1 / \phi^{\prime}\right\|_{\infty}$.

Proof. Without loss of generality we can assume that $\phi$ is an increasing function. Let $C_{\phi}$ be a composition operator on $L^{p}(\mu)$, and let $x, y \in R$ such that $x<y$. Then since the inverse image of the interval $[\phi(x), \phi(y)]$ under $\phi$ contains the interval $[x, y]$, we can conclude by applying $C_{\phi}$ to the characteristic function of $[\phi(x), \phi(y)]$ and calculating the norms that

or equivalently

$$
\left\|C_{\phi}\right\|^{p}(\phi(y)-\phi(x)) \geqslant y-x
$$

$$
(\phi(y)-\phi(x)) /(y-x) \geqslant 1 /\left\|C_{\phi}\right\|^{p} .
$$

Since $x, y$ are chosen arbitrarily, it follows that

$$
\left\|1 / \phi^{\prime}\right\|_{\infty} \leqslant\left\|C_{\phi}\right\|^{p}<\infty .
$$

Conversely, suppose $1 / \phi^{\prime}$ is essentially bounded. Then there is an $m>0$ such that $\phi^{\prime}(x)>1 / m$ (a.e.). We can write $\phi(x)=\phi_{a}(x)+\phi_{s}(x)$, where $\phi_{a}$ is absolutely continuous and $\phi_{s}$ is the singular part of $\phi$. Let $x, y \in R$ and let $x<y$. Then

$$
\phi(y)-\phi(x) \geqslant \phi_{a}(y)-\phi_{a}(x)=\int_{x}^{y} \phi_{a}^{\prime} d \mu \geqslant(y-x) / m
$$


and hence

$$
y-x \leqslant m[\phi(y)-\phi(x)] .
$$

Let $E=[c, d]$ be a half-open interval. Let

$$
\beta=\inf \{x: \phi(x) \geqslant c\} \text { and } \gamma=\sup \{y: \phi(y) \leqslant d\} .
$$

Then it follows from ( $*$ that $\beta>-\infty$ and $\gamma<\infty$. We claim that $\beta \leqslant \gamma$ and $\phi^{-1}(E) \subset[\beta, \gamma]$. Suppose $\beta>\gamma$. Then there exists an $x$ such that $\beta>x>$ $\gamma$. From this and the definitions of $\beta$ and $\gamma$ it follows that $d<\phi(x)<c$ which is a contradiction to the fact that $c \leqslant d$. Hence $\beta \leqslant \gamma$. To prove that $\phi^{-1}(E) \subset[\beta, \gamma]$, let $x \in \phi^{-1}(E)$. Then $\phi(x) \in E$ and hence $c \leqslant \phi(x)<d$. Since $\phi(x) \geqslant c$, we obtain that $\beta \leqslant x$ and since $\phi(x) \leqslant d$ we get $x \leqslant \gamma$. Thus $\beta \leqslant x \leqslant \gamma$. This proves our claim. In case $\beta=\gamma$, the inequality $\mu \phi^{-1}(E) \leqslant$ $m \lambda(E)$ follows trivially. If $\beta<\gamma$, then let $x, y \in R$ such that $\beta<x<y<$ $\gamma$. The the interval $[\phi(x), \phi(y)] \subset E$. From inequality (*) it follows that

$$
y-x \leqslant m[\phi(y)-\phi(x)] \leqslant m \mu(E)
$$

since $x, y$ are chosen arbitrarily, we can conclude that

$$
\mu \phi^{-1}(E) \leqslant m \mu(E) .
$$

Thus the above inequality holds for every half-open interval. The class of sets for which the above inequality holds is a monotone class containing the ring of finite unions of half-open intervals. By Theorem $A$ of $[1, p .27]$ the above inequality holds for all Borel sets. Hence, by Theorem $1, C_{\phi}$ is a composition operator. This proves the theorem.

2. Polynomials and composition operators. In ths section we assume that $\phi$ is a real polynomial. Nonvanishing of the derivative of the function is a necessary condition for the inducibility of the composition operator as we have seen in Theorem 2. It turns out that this condition is also sufficient in the case of polynomials. This we shall show in the following theorem.

THEOREM 4. Let $\phi$ be a polynomial with the real coefficients. Then $C_{\phi}$ is a composition operator on $L^{p}(\mu)$ iff $\phi^{\prime} \neq 0$. Furthermore, if $\phi^{\prime} \neq 0$, then $\left\|C_{\phi}\right\|^{p}$ $=\sup \left|1 / \phi^{\prime}\right|$.

Proof. The proof of the 'only if' part is evident from Theorem 2. To prove the 'if' part suppose $\phi^{\prime} \neq 0$. Then since $\phi$ is a polynomial $1 / \phi^{\prime}$ is bounded and also $\phi$ is a monotone function. Hence, by Theorem $3, C_{\phi}$ is a composition operator and $\left\|C_{\phi}\right\|^{p}=\left\|1 / \phi^{\prime}\right\|_{\infty}=\sup \left|1 / \phi^{\prime}\right|$. This proves the theorem.

Corollary 1. A necessary condition that $C_{\phi}$ be a composition operator on $L^{p}(\mu)$ is that the degree of $\phi$ be not even.

Proof. If degree of $\phi$ is even, then $\phi$ is zero at some point. Hence, by Theorem $4, C_{\phi}$ is not bounded composition operator.

COROllary 2. Let $\phi(x)=a x^{3}+b x^{2}+c x+d$, where $a, b, c, d \in R$. Then $C_{\phi}$ is composition operator on $L^{p}(\mu)$ iff $b^{2}<3 a c$.

3. Rational functions and composition operators. Let $\omega=\phi / \psi$ be a rational function, where $\phi$ and $\psi$ are polynomials with real coefficients. We assume 
that $\phi$ and $\psi$ have no common real zeros, and the coefficients of the leading terms of $\phi$ and $\psi$ are equal to 1 . We also assume that $\operatorname{deg} \psi>1$, where by deg $\psi$ we mean the degree of $\psi$. In $\$ 2$ we have proved that nonvanishing of the derivative of a polynomial is a necessary and sufficient condition for the inducibility of the corresponding composition operator on $L^{p}(\mu)$. But in case of rational functions a little more is needed which will be investigated in this section.

TheOREM 5. Let $\omega=\phi / \psi$ be a rational function. Then $C_{\omega}$ is a composition operator on $L^{p}(\mu)$ implies that $\operatorname{deg} \phi>\operatorname{deg} \psi$.

Proof. If $\operatorname{deg} \phi \leqslant \operatorname{deg} \psi$, then $\omega$ is bounded on a set of infinite measure and hence, by Theorem $1, C_{\omega}$ is not a composition operator.

THEOREM 6. Let $\omega$ be a rational function. Then $C_{\omega}$ is a composition operator on $L^{p}(\mu)$ implies that $\omega$ has at least one real zero.

Proof. Suppose $\omega$ does not have any real zeros. Then in case $\omega$ is continuous there will be at least two points $x_{1}$ and $x_{2}$ such that $\omega\left(x_{1}\right)=\omega\left(x_{2}\right)$. Applying the mean value theorem we can conclude that $\omega^{\prime}\left(x_{0}\right)=0$ for some $x_{0}$ such that $x_{1}<x_{0}<x_{2}$. Hence, by Theorem 2, $C_{\omega}$ is not a composition operator. In the case $\omega$ is discontinuous, it must have at least two poles. Taking any two consecutive poles and applying the mean value theorem, we can again conclude that $\omega^{\prime}$ is zero at some point. Hence $C_{\omega}$ is not a composition operator. This proves the theorem.

COROllaRY 3. Let $\omega=\phi / \psi$ be a rational function. Then $C_{\omega}$ is a composition operator implies that $m-n$ is not an even integer, where $m=\operatorname{deg} \phi$ and $n=\operatorname{deg} \phi$.

Corollary 4. Let $\omega$ be a continuous rational function. Then $C_{\omega}$ is a composition operator only if $\omega$ has one and only one real zero.

Now we shall need the following lemma.

Lemma 1. Let $\omega=\phi / \psi$ be a discontinuous rational function and let $m$ and $n$ be degrees of $\phi$ and $\psi$ respectively. Then $m>n$ and $\omega^{\prime} \neq 0$ implies that $C(T)=C(Z)$ and poles and zeros of $\omega$ occur alternately on $R$, where $C(Z)$ and $C(T)$ denote the number of distinct real zeros and number of distinct real poles of $\omega$ respectively.

Proof. If $C(Z)>C(T)$ or $C(Z)<C(T)$, then an application of Rolle's theorem in the first case and of the mean value theorem in the second case gives one point where $\omega^{\prime}$ is zero, which is a contradiction to hypothesis. If poles and zeros do not occur alternately, then we can conclude that $\omega^{\prime}$ is zero at some point and this is again a contradiction. Thus the proof of the lemma is complete.

Now we shall prove the main theorem of this section.

TheOREM 7. Let $\omega=\phi / \psi$ be a rational function. Then $C_{\phi}$ is a composition operator on $L^{p}(\mu)$ iff $\operatorname{deg} \phi>\operatorname{deg} \psi$ and $\omega^{\prime} \neq 0$ at every point of $R$ where it exists. 
Proof. The 'only if' part is clear from Theorems 2 and 3. To prove the 'if' part suppose $\operatorname{deg} \phi>\operatorname{deg} \psi$ and $\omega^{\prime}$ is not zero. Then in the case $\omega$ is continuous $\omega^{\prime}$ is also continuous and $1 / \omega^{\prime}$ is a bounded function. Hence, by Theorem $3, C_{\omega}$ is a composition operator. If $\omega$ is discontinuous, then from Lemma $1, C(Z)=C(T)$ and zeros and poles occur alternately. This implies that $\omega$ is monotone on the interval starting and ending at the consecutive poles. Thus it can be shown $[1$, p. 136] that

$$
\left(d \mu \omega^{-1} / d \mu\right)(x)=\sum_{y \in \omega^{-1}(\{x\})} 1 / \omega^{\prime}(y) .
$$

Since $\omega^{\prime} \neq 0$, the Radon-Nikodým derivative $d \mu \omega^{-1} / d \mu$ of the measure $\mu \omega^{-1}$ is essentially bounded. Hence, by Theorem $1, C_{\omega}$ is a composition operator on $L^{p}(\mu)$. This completes the proof of the theorem.

\section{REFERENCES}

1. P. R. Halmos, Measure theory, Van Nostrand, Princeton, N. J., 1950. MR 11, 504.

2. W. C. Ridge, Spectrum of a composition operator, Proc. Amer. Math. Soc. 37 (1973), 121-127. MR 46 \#5583.

3. Characterization of abstract composition operators, Proc. Amer. Math. Soc. 45 (1974), 393-396. MR 49 \#11310.

4. R. K. Singh, Compact and quasinormal composition operators, Proc. Amer. Math. Soc. 45 (1974), 80-82. MR 50 \# 1043.

5. , Normal and Hermitian composition operators, Proc. Amer. Math. Soc. 47 (1975), $348-350$.

$6 . \quad$ Invertible composition operators on $L^{2}(\lambda)$, Proc. Amer. Math. Soc. 56 (1976), 127-129.

Department of Mathematics, University of Jammu, Jammu (TAWi), India 